\title{
El congreso virtual de la sociedad interamericana de psicología 2020: aportes de la psicología ante la covid-19
}

\author{
Angélica María Lechuga Quiñones ${ }^{1}{ }^{2}$ \\ Universidad Juárez del Estado de Durango, Durango, México.
}

\section{RESEÑA DEL LIBRO}

Luna-Sánchez, S. E., Pérez-Acosta, A. M., Samaniego-Pinho, A., Vergara-Morales, J. E. (compiladores) (2020). Memorias del Congreso Virtual de la Sociedad Interamericana de Psicología 2020: Aportes de la Psicología ante la Covid-19. Sociedad Interamericana de Psicología y Universidad Carlos Albizu. DOI: https://doi.org/10.30849/CIP.V.2020.Memorias.

El Primer Congreso Virtual de la Sociedad Interamericana de Psicología 2020: Aportes de la Psicología ante la COVID-19, sin duda, fue un gran reto de organización, de la Sociedad Interamericana Psicología y Universidad Carlos Albizu. Representó, un esfuerzo, ya que implicó contar con información sobre los efectos que la pandemia del COVID-19 ha provocado, o se han manifestado hasta el momento en la salud mental y el bienestar de la población en el mundo.

Fue un evento planificado como un foro de intercambio de conocimientos sobre una sola problemática: la pandemia del COVID-19, por lo que dar cuenta de los conocimientos científicos y las formas de intervención generadas por la psicología para enfrentar las secuelas asociadas a la pandemia del Coronavirus fue el objetivo principal del Congreso.

El evento se llevó a cabo los días 21, 22 y 23 de octubre de 2020, y además tuvo como marco la conmemoración de la vida y obra del Dr. Reynaldo Alarcón Napuri, (1924-2020) y la Dra. Isabel Reyes Lagunes (1942-2020).

El Primer Congreso Virtual de Psicología, ha quedado documentado en un libro de memorias, el cual, se puede visualizar como histórico. Quienes estuvieron a cargo de éste trabajo de compilación de gran importancia fueron: Andrés M. Pérez-Acosta, Sandra

\footnotetext{
1 Correspondence about this article should be addressed Angélica María Lechuga Quiñones: anmalequi@gmail.com 2 Conflicts of Interest: The authors declare that the research was conducted in the absence of any commercial or financial relationships that could be construed as a potential conflict of interest.
} 
Elizabeth Luna-Sánchez, Antonio Samaniego-Pinho y Jorge Vergara-Morales, integrantes del Comité Científico propuestos por La Sociedad Interamericana de Psicología 2020.

El esfuerzo realizado por este grupo es realmente meritorio, no sólo por lo que implico metodológicamente tener un gran eco de participación a nivel Latinoamericano y Europa, con estudiantes y profesionales de la psicología de diferentes países, recibiendo una gran cantidad de trabajos que integran este libro, si no por el involucramiento anímico que seguramente experimentaron al recibir el contenido de los resúmenes, cargados de innumerables experiencias y comentarios con grandes matices de vulnerabilidad, escritos posiblemente, llenos de energía inquietante y con sentimientos encontrados por parte de todos los involucrados ante la situación de pandemia.

Sin duda, éste evento, plasmado en las memorias del Primer Congreso virtual de la SIP, se llevó a cabo con gran actitud positiva por parte de mucha gente de diferentes partes del mundo, con una idea en común, aportar en estos tiempos de dificultad.

También, es importante reconocer la valentía de quienes participaron de diferentes maneras en este evento, ya que quizá, muchos, tuvieron que afrontar situaciones de pérdida y dolor, dificultades para ofrecer sus servicios y/o para obtener información, arriesgando su salud, con la firme convicción de participar desde diferentes posiciones en éste Primer Congreso virtual de la SIP.

El libro de memorias, de éste gran evento, se ha hecho con gran cuidado desde su portada (véase Figura 1). El autor, Sady Ariel Becerra Muñoz la ha titulado: "El florecimiento de la quila", la cual, ha servido como fuente de inspiración para el poeta y ensayista Elicura Chihuailaf, 2020, quién en la contraportada nos comparte una descripción muy sentida, y con gran simbolismo a propósito de este encuentro; “[En el] destape de la pandemia [...] Lo primero fue recordar que en nuestro Wallmapu habían florecido las quilas lo que, en la memoria mapuche, y del Chile profundo, significa tiempos de padecimiento [...] ha llegado el tiempo de escuchar de verdad a nuestros Álter Nativos que siempre estuvieron, están y estarán en diálogo con la Naturaleza, asumiéndose como iguales en las diversidades de ser hijos e hijas de la Madre Tierra y del Universo, ni más ni menos que todos los seres vivos y aquellos aparentemente inanimados. Y que sean los y las mejores representantes de nuestros Álter Nativos los que conformen Consejos que lideren y gobiernen, para un Buen Vivir, a los diversos territorios del mundo". 


\section{Figura 1}

"El florecimiento de la quila"

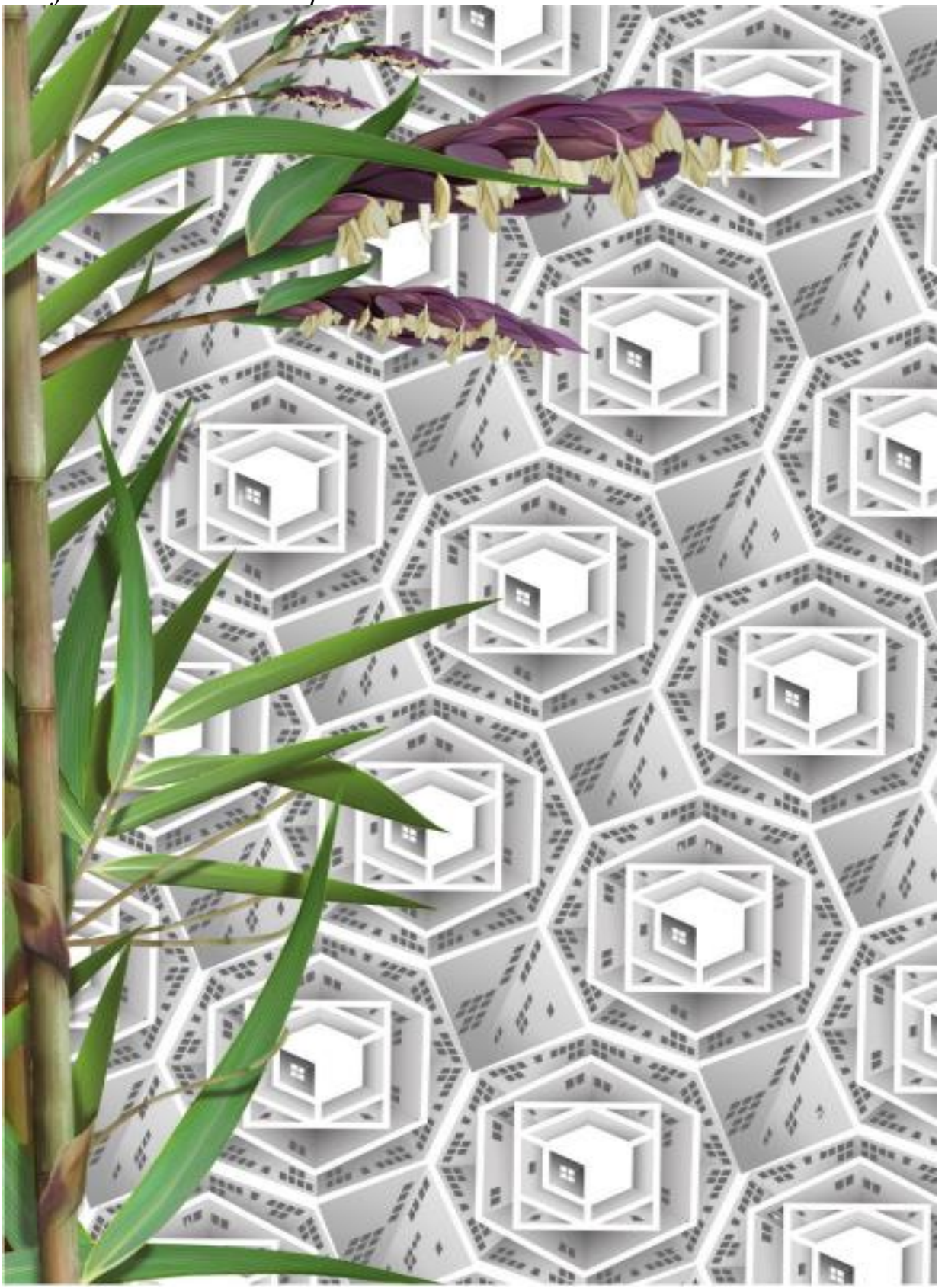

Nota: Tomado de Memorias del Congreso Virtual de la Sociedad Interamericana de Psicología 2020: Aportes de la psicología ante la COVID-19, S. E. Luna-Sánchez, A. M. Pérez-Acosta, A. Samaniego-Pinho, J. E. Vergara-Morales (Comp.), 2020, SIP \& Universidad Carlos Albizu. 
Estas memorias representan una gran compilación, la cual muestra uno de los mayores desafíos que ha enfrentado el mundo en este siglo que es la pandemia por COVID-19, exigiendo acción, cooperación y solidaridad en todos los niveles, con mayores exigencias en el desarrollo de las capacidades humanas y un involucramiento directo en los diferentes aspectos del conocimiento, con implicaciones directas desde el punto de vista biológico, económico, político, tecnológico y educativos, entre otros, así como situaciones tan finas que seguramente dejaran huellas de por vida en el contexto mundial. En éste sentido, una de las cosas que realmente han sido desafiantes de la pandemia COVID-19 para todas las edades, razas y géneros, es la "ruptura de la normalidad", tal como lo menciona en el prólogo de estas memorias la Alta Comisionada de la ONU para los Derechos Humanos Michelle Bachelet, destacando también que éste fenómeno ha exigido en todos los niveles, una reflexión crítica sobre la importancia de conservar y preservar la salud.

También ha sido evidente la necesidad y sobre exigencia del personal de salud, principalmente, justo por la urgencia de atender desde lo individual hasta lo colectivo, de correr sobre el tiempo para ofrecer alternativas de atención (saturación en los servicios de salud), como de cura (elaboración de una vacuna).

La Psicología, en este sentido, como parte de los servicios de cuidar y preservar la salud, es de gran importancia, por ejemplo: ante la contingencia sanitaria se crearon múltiples respuestas innovadoras, muchas de las cuales se basaron en la utilización de las TIC para afrontar las consecuencias sobre la salud mental de la población. Otras tantas acciones realizadas por la Psicología han quedado documentadas en el libro de memorias del Congreso Virtual de la SIP 2020.

Sin duda la Psicología ha estado jugado un papel importante para la sociedad, durante la historia, avanzado constantemente, teniendo un papel relevante y necesario, sobre todo en éste tiempo de pandemia. La Psicología en éste tiempo de crisis, ha tratado de entender, orientar, así como ofrecer posibles respuestas y/o soluciones a las necesidades que se han presentado y, seguirán emergiendo como respuesta de las innumerables experiencias en el ámbito psicosocial, en esto que se ha llamado "Nueva Anormalidad" como una de las expresiones propias de la histórica pandemia del COVID19.

Este Congreso Virtual, de acuerdo a lo plasmado en sus memorias, logró tener una gran respuesta, a pesar de la incertidumbre experimentada en éste tiempo. El Comité Científico convocó a profesionales, científicos, profesores y estudiantes de la disciplina 
y profesión psicológica a remitir sus contribuciones, y como punto de encuentro, sería la modalidad virtual para intercambiar los conocimientos y las experiencias generadas ante la problemática de la pandemia por el COVID-19 en sus diferentes regiones.

Se recibieron y evaluaron más de 400 propuestas entre las diferentes modalidades de participación. 385 resúmenes fueron aceptados, y presentados 345. Participaron 25 países: Argentina, Bélgica, Brasil, Canadá, Chile, Colombia, Costa Rica, Cuba, Ecuador. El Salvador. España, Estados Unidos de América, Francia, Guatemala, Honduras, Italia, México, Nicaragua, Panamá, Paraguay, Perú, Puerto Rico, Suiza, Uruguay, y Venezuela.

Se identificaron una gran variedad de áreas temática: Psicología social, comunitaria y política, Salud y bienestar humano, Psicología de la salud, clínica y hospitalaria, Educación, subjetividad y desarrollo humano, Psicología de las emergencias, catástrofes y desastres, La psicología del trabajo y las organizaciones, y Evaluación y psicodiagnóstico, Estudios de género, Psicología del desarrollo y ciclo vital, Neuropsicología, neurociencias y psicología cognitiva, Pareja, sexualidad y diversidades sexuales, Epistemología, metodología e historia de la psicología, Psicología y discapacidad, Adicciones y consumos problemáticos, Psicogerontología, Psicología del deporte, Psicología experimental y comparada, Psicología jurídica y forense, Ética y deontología profesional, Psicología ambiental, y Psicología y migraciones.

En cuanto a temas presentados, el $88 \%$ correspondieron a Reportes de investigaciones, Relatos de experiencias, Estudios preliminares, y Modelos intervenciones. El 12\% restante, fueron contribuciones orientadas a: diagnósticos, dispositivos institucionales, casuísticas, informes técnicos, entre otros.

En el libro de memorias se documenta con gran detalle como estuvo determinada la organización del congreso. Se plantearon dos modalidades: sincrónica y asincrónica, las cuáles, se describen con gran detalle; demás, se cuenta con la evaluación de los participantes por cada área: caracterización de los participantes, grado académico, distribución por Grupo Etario. También se hicieron evaluaciones de acuerdo a la distribución de quienes se inscribieron y estuvieron presentes en los Talleres Pre y Pos Congreso.

En las memorias de éste Primer Congreso Virtual de la Sociedad Interamericana de Psicología 2020: Aportes de la Psicología ante la COVID-19, ha quedado evidenciado con gran detalle las actividades que tuvieron lugar durante los días 21, 22 y 23 de octubre. Tanto aquellas que se desarrollaron de manera sincrónica, como las protocolares de apertura y cierre, los homenajes al Dr. Reynaldo Alarcón y la Dra. Isabel Reyes Lagunes, 
y la entrega de los premios correspondientes a su remembranza. Así mismo, las conferencias por invitación que tuvieron lugar por distinguidos especialistas, como la conferencia de apertura de la Dra. Michelle Bachelet (Alta Comisionada de la ONU para los Derechos Humanos), de igual manera los diferentes simposios, mesas redondas, y las presentaciones de publicaciones. Todas éstas actividades se transmitieron en vivo, en diferentes salas simultáneas y la mayoría fueron grabadas para su posterior reproducción y difusión.

Se presentaron 22 mesas redondas/conversatorios, 14 talleres pre y post-congreso, 12 simposios temáticos, 11 conferencias por invitación, 9 simposios institucionales, 3 simposios por invitación y 3 presentaciones de libros. De forma asincrónica se presentaron 183 trabajos libres, 42 pósters/carteles y 5 foros especiales los cuales fueron agrupados en diferentes Foros de Debates y se les pidió a los correspondientes autores la grabación de sus exposiciones, éstos foros se abrieron durante un tiempo determinado y las/los coordinadores/es designadas/os tuvieron la tarea de plantear ejes articuladores y preguntas transversales. En consecuencia, esta modalidad fue asincrónica y cada expositor tuvo la posibilidad de dar a conocer su trabajo en los diferentes foros y los participantes pudieron expresar sus comentarios y preguntas.

Dadas las condiciones del evento se provecharon al máximo los recursos tecnológicos para lograr un mayor alcance de los conocimientos y las experiencias obtenidas. Aunado a esto, se planteó la posibilidad de realizar webinars posteriores al congreso que permitan el encuentro virtual de los expositores relativos a los diferentes Foros de Debates, ya que identificó que muchos de los/las participantes entraron en contacto por primera vez con éste tipo de encuentros y seguramente darán otro tipo de interacciones dada la comunicación y los datos plasmados en estas memorias que les pudieran interesar algunas de las temáticas que están trabajando los colegas en sus lugares o bien en otras localidades del mundo.

Uno de los aspectos a destacar de manera importante en libro de memorias, es el dedicarle un espacio a mencionar y reconocer de manera muy importante el liderazgo que han tenido en la Sociedad Interamericana de Psicología, inicialmente, y durante mucho tiempo los hombres, y posteriormente ha sido evidente el gran trabajo desarrollado por las mujeres, quienes han generado cambios en la gran temática de psicología con población mayoritariamente femenina. Por ejemplo: en los programas científicos de los congresos interamericanos y regionales se ha visto que están cargados de mujeres investigadoras, interventoras que han sometido sus trabajos y son quienes han nutrido en 
gran medida el conocimiento en estos eventos, por ejemplo: según la evaluación del Congreso virtual de la SIP 2020 mostró que 69\% de las participantes fueron mujeres, y el $30 \%$ hombres. A propósito de esto, fue que, durante el planteamiento de este Congreso, se propuso un nuevo premio, el cual visibilizara el trabajo realizado por profesionales, estudiantes e instituciones en la emergencia de la COVID-19. El nombre que recibió este premio se otorgó a una mujer, a quien, entre sus múltiples reconocimientos, nacionales e internacionales, se destaca el Premio Interamericano de Psicología en 2007. Ella contribuyó con el desarrollo de la psicología, en áreas como cultura y personalidad, etnopsicometría y psicología política y fue la primera presidenta de la SIP, la Dra. Isabel Reyes Lagunes. La participación a la que se convocó fue en las categorías profesional, estudiantil e institucional. En el evento se hizo un homenaje a ésta gran psicólogaInvestigadora Mexicana para recordar sus aportes a la psicología y a la organización en su período de gobierno y durante todo su trayecto como miembro muy activa.

También se llevó a cabo el homenaje al Dr. Reynaldo Alarcón Napuri, máximo representante de la psicología peruana en áreas como la Psicometría y la psicología de la pobreza, psicología positiva, en particular Psicología de la Felicidad y Etnopsicología. El Dr. Alarcón cuenta con una trayectoria nacional e internacional, se hizo acreedor al Premio Nacional de Cultura y al premio Rubén Ardilla a la Investigación Científica en Psicología, otorgado por la SIP en 2015. Este investigador fue un importante socio de la Sociedad Interamericana de Psicología.

Finalmente se puede decir que en estas memorias queda documentado el valioso y gran trabajo realizado por los diferentes comités de la SIP. Se agradece a cada uno/una de los/las colegas, estudiantes hombres y mujeres que sumaron 52, y han representado con su gran apoyo a Costa Rica, Colombia, México, Perú, Chile, Argentina, Brasil, Puerto Rico, República Dominicana, Honduras y Guatemala.

Se agradece a las instituciones que se han sentido convocadas por esta iniciativa de la (SIP). Y en especial a los miembros que siguen activos en ésta sociedad, a aquellos que forjaron un compromiso en los Grupos de Trabajos y a los Representantes Nacionales, que se involucraron en la gestión del evento y la participación con el envío de sus propuestas.

Es importante decir, que, en este libro de memorias se evidencia el gran compromiso y dedicación a quién se nombró para representar al comité científico, el Dr. Andrés Pérez-Acosta quien invitó a la Dra. Sandra Elizabeth Luna-Sánchez para Codirigir el Comité Científico y al Mg. Antonio Samaniego Pinho y el Dr. Jorge Vergara 
Morales quienes lograron resaltar el liderazgo femenino, lo cual tuvo eco en su cocoordinadora y se constituyó todo el Comité Científico Internacional por mujeres Doctoras de países de las Américas. Se nombraron veinte doctoras en psicología de veinte países de las Américas, que tuvieron a su cargo la construcción conjunta del programa científico y la evaluación de los premios que se otorgaron.

Otro de los logros de éste evento y derivado del gran trabajo del comité Científico es que adicionalmente se propuso la convocatoria para un libro compilado por la editorial Springer con los trabajos completos que se presentaron y fueron seleccionados por un comité revisor especial.

En éste documento se denota la gran entrega y liderazgo con la que el presidente Dr. Carlos Zalaquett, a la mano del comité Organizador, científico, de Difusión, Científico Internacional y el de finanzas lograron llevar a buen fin, este gran e histórico evento, que en palabras del Coordinador del Comité Organizador del Congreso Virtual de la SIP 2020 Dr. Miguel Gallegos, resalta la participación y observándose en ella, gran pasión en el trabajo que realizaron todos(as) los participantes, entre los que se encuentra el realizado por 108 Evaluadores (as) de las propuestas sometidas al Congreso Virtual de la SIP 2020.

Sin duda, hay que reconocer el valioso material hay en que éste libro de memorias el cual puede constituirse en un acervo de conocimientos de diferentes experiencias, investigaciones y producciones ligadas a la pandemia por el COVID-19, la cual formará parte de la historia, en la que se vieron involucradas diferentes disciplinas del conocimiento de la psicología de los diferentes 25 países hablando de un tema en común: COVID-19. 